\title{
Constructing Knowledge in an Interactive Game-Like Design Environment for STEM Education
}

\author{
Gayatri Mehta, Jeanne Tunks, Kiran Sanagapaty, Alok Pal, and Rani Deepika Balavendran Joseph
}

\begin{abstract}
In this study, we examine how the upper and lower level performers construct their knowledge in an open-ended interactive challenging game-like design environment. The interactive game UNTANGLED was used for this study. Piaget's theory of knowledge construction was used to examine performance data. Findings showed that the upper performers could be characterized as constructivist learners who are accommodators, learners who alter schema based on new information or experiences. These players used various incentives to both achieve success in completion of the game puzzles and change perspective during the game play. In contrast, the lower level performers used a constructed knowledge adaptation schema of assimilation. They did not complete the game puzzles and were not motivated by any incentives in the game play. Discussion and implications suggest how classroom teachers or game designers, who design games with an educational purpose, can recognize the needs of both constructors of knowledge: assimilators and accommodators and adjust instruction to assure success for both types of knowledge constructors.
\end{abstract}

Index Terms-Engineering education, games with a purpose, mapping, placement, constructive learning, STEM games.

\section{INTRODUCTION}

\section{A. Constructivism}

Learning, the purposeful outcome of education takes on multiple forms and is best observed when learners construct their own knowledge based on experiences. In a learning world filled with technology, both in and out of the classroom, learners have extensive experiential opportunities that can lead to constructed knowledge. The most prominent theory of constructivism purported by Jean Piaget, asserts that experiential learning requires adaptation and occurs as either assimilation or accommodation [1]. In the former, a learner operates from a personal framework of knowledge and incorporates new learning experiences, without changing the original framework. In the latter, the learner reframes the original mental framework based on new information gained from experiential learning opportunities.

For this study, constructivist theory was applied, post-priori, using an inductive approach to examine and

Manuscript received June 13, 2016; revised August 30, 2016. This work was supported in part by the National Science Foundation under grants CCF-1117800 and CCF-1218656.

Gayatri Mehta is with Electrical Engineering, University of North Texas, Texas, USA (e-mail: gayatri.mehta@unt.edu).

Jeanne Tunks, Kiran Sanagapaty, Alok Pal, and Rani Deepika Balavendran Joseph are with College of Education, University of North Texas, Texas, USA. (e-mail: jeanne.tunks@unt.edu, KiranSanagpaty@my.unt.edu,

RaniDeepikaBalavendranJoseph@my.unt.edu). define the findings. Data, collected as self-selected, random players engaged with the puzzles, were examined through the lens of Piaget's constructivist theory. The use of post-priori data, coupled with an inductive approach to constructivist theory, lead to the assumptions applied to the solutions to the puzzles. Assumptions were made about how learners constructed their knowledge, either as assimilators or accommodators.

\section{B. Gaming and Constructivism}

Electronic game developers have applied constructivist theory to create meaningful educational learning experiences for gamers [2]-[9]. Findings from studies of game design and the use of constructivist principles to design games yielded stronger games that had greater educational value and meaning for the players, affirming the position posited by Piaget. Gaming researchers examined the effective use of constructivist theory and found that game design was enhanced and learning more apparent when the theory was applied [9]-[13].

In addition to the value of the use of the theory of constructivism for gaming design purposes, instruction with games central to pedagogical practices resulted in higher levels of learning [6], [12], [14]-[20]. Researchers noted that instructional games, when supported by instructional practices using constructivism, netted increased learning across multiple instructional settings, including nursing, social studies, and science. The connection between gaming as instrumental in instruction, when constructivist theory is applied affirms the validity of applying the theory.

Although the application of the theory for instructional purposes validates the use of the theory, the theory was originally designed to support learning. Other researchers (Delia, 1977; Gholson \& Craig, 2006; Giustini, 2009; Hoge \& Hughes, 2010; Mansour \& El-Said, 2009; Moore et al., 2014; Morgan \& And, 1997; Pascarella, 2008; Razak \& Connolly, 2013; Saadé \& Kira, 2004; Vu Minh, 2007) found that learning was enhanced in gaming environments when design and constructivist theory were combined [5], [11], [13], [19]-[26]. Students reported deeper understanding, meaningful learning, and advances in knowledge when games were presented with constructivist principles applied. However, gaps in presentation of learning regarding the direct application of Piaget's principles of accommodation and assimilation remained.

Several studies examined the process of learning, in the form of decision-making, an important aspect of constructed learning. Higher levels of success were noted on AI games when the halo effect of an interactive computer-aided tool used prior to testing was used by one group, when compared to another that did not have access to the same [20]. Hoge and 
Hughes noted that when secondary students played micromanagement computer games, based on NAEP guidelines in an educational setting, learners working in teams, enhanced students' understanding of complex science and mathematics concepts [5]. The games encouraged students to seek advanced knowledge of content, supporting constructivist problem based learning.

\section{Phenomena}

Although the original purpose of the interactive game-like design environment project was to create an electrical engineering game with a purpose, to determine anonymous players' solutions to the game, resulting in algorithms that could lead to new technologies, the purpose took on different trajectory when it phenomenologically emerged through an examination of the data from the game play toward a study of educational purposes. An alternate examination of the data generated the notion that individual players constructed their own knowledge

\section{Purpose}

The purpose of this study was to answer the following research question: How do upper and lower level performers construct their knowledge in an open-ended interactive challenging game-like design environment for STEM education?

\section{E. Hypothesis}

It was hypothesized that upper and lower level performers would construct their differently from each other, but there was no prediction as to whether either would adapt as assimilators, accommodators, or a combination thereof.

\section{BACKGROUND}

Scientific games or Games with a Purpose have recently gained much attention as more and more researchers / scientists are harnessing the knowledge of ordinary citizens to solve many of their research problems [27]-[33]. More information about how crowds solve problems can be found in books [34]-[36]. In our study, we used UNTANGLED, which is an interactive gaming environment to uncover human mapping / placement strategies. It was designed to harness human intelligence and discover new mapping techniques for low power portable electronics. The game has been online since May 2012 and has attracted more than 900 players till date. Players used random user names or self-selected user names to play puzzles. Information about the players such as their background, demographics, age etc. was not saved in the database. The only information that has been considered is how players' solve puzzles. Our study was determined to qualify for an exemption from the Institutional Review Board of our university. The game is broadly accessible to anyone and players do not need to have special engineering background to play this game. There are in-depth tutorials in the game that provide good hands-on experience to the players. There are several sub-games in UNTANGLED and each sub-game has puzzles arranged according to the level of difficulty. To motivate players, incentives are given to players in terms of badges or medals. The game has a leaderboard where players can check their standings. The game is available at https://untangled.unt.edu and more details about the game can be found in [37].

The contribution of the current manuscript is to study how the upper and lower level players construct their knowledge in an open-ended interactive challenging game-like design environment.

\section{EXPERIMENTAL SET-UP}

This section describes our experimental set-up and methods that were used to conduct the study presented in this paper. The experimental protocol for all studies was determined to qualify for an exemption from the Institutional Review Board of the University of North Texas. IRB protocols were followed in all cases.

In this paper, we present results for three games: 4Way2Hops, 4Way1Hop, and 8Way, shown in Figure 1. 4Way2Hop is a game that allows horizontal and vertical connections that skip two nodes. 4Way1Hop is a game allowing connectivity to direct horizontal and vertical neighbors, as well as horizontal and vertical connections that skip one node. $8 \mathrm{Way}$ is a game where blocks can connect to any of their 8 neighbors.

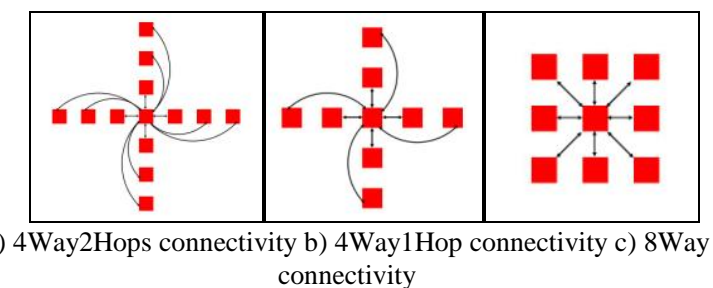

Fig. 1. 4Way2Hops, 4Way1Hop, and 8Way mesh architectures.

The seven benchmarks considered for our study are chosen from the domain of signal and image processing. The benchmarks include Sobel (E1), Laplace (E2), GSM (E3), ADPCM decoder (M1), ADPCM encoder (M2), IDCT row (H1), and IDCT col (H2) benchmarks. Basic statistics are shown in Table I. The benchmarks are arranged in the game according to the difficulty level and can be categorized into easy, medium and hard levels.

TABLE I: BASIC INFORMATION RELATED TO THE BENCHMARKS

\begin{tabular}{lccccccc}
\multicolumn{1}{c}{ Levels } & E1 & E2 & E3 & M1 & M2 & H1 & H2 \\
\hline Blocks & 24 & 29 & 29 & 29 & 36 & 52 & 61 \\
Edges & 29 & 29 & 34 & 36 & 53 & 63 & 72 \\
\hline
\end{tabular}

UNTANGLED game was released in May 2012 and has been online since then. An online gaming competition was conducted in 2012 at worldwide level and it lasted for ten days from August 10 to August 20. After the competition, the game has remained online and continued to attract new and returning players. Several resources such as online press releases and university-sanctioned posts on social networking websites were used to advertise about the competition. Gift cards were given to the winners of each game and the overall competition. Rankings were visible on the UNTANGLED leaderboard throughout the competition and can still be viewed on the game website https://untangled.unt.edu/competition/home.php. The game 
has attracted more than 900 players who have generated more than 12000 solutions till date. In the results reported here, we used all played games, including those contributed after the end of the competition. In each case, we considered top 20 and bottom 20 players.

\section{DATA ANALYSIS AND FINDINGS}

Independent-samples t-test were conducted to compare scores, violations, types of moves, quarters of puzzle construction, and difficulty of puzzles in lower and upper performers' performances on the puzzles.

\section{A. Scores and Violations}

There was a significant difference in the scores for lower performers $(\mathrm{M}=68591.93, \quad \mathrm{SD}=130728)$ and upper performers $(\mathrm{M}=270404.54, \mathrm{SD}=218258)$ as they solved the puzzles; $\mathrm{t}(20)=-4.224, \mathrm{p}=.000$. There was a significant difference in the number of violations for lower performers $(\mathrm{M}=7.5, \mathrm{SD}=5.963)$ and upper performers $(\mathrm{M}=0, \mathrm{SD}=0)$ as they solved the puzzles; $\mathrm{t}(20)=-5.762, \mathrm{p}=.000$. The results suggest that the upper level performers were using different constructivist skills when compared to lower performers.

\section{B. Types of Moves}

There was a significant difference in the number of single moves for lower performers $(\mathrm{M}=29.57, \mathrm{SD}=13.69)$ and upper performers $(\mathrm{M}=48.43, \mathrm{SD}=27.43)$ as they solved the puzzles; $t(35)=-6.776, p=.000$. There was a significant difference in the number of multi moves for lower performers $(\mathrm{M}=2.44$, $\mathrm{SD}=1.01)$ and upper performers $(\mathrm{M}=3.57, \mathrm{SD}=2.55)$ as they solved the puzzles; $\mathrm{t}(35)=-3.396, \mathrm{p}=.002$. There was a significant difference in the number of swap moves for lower performers $(\mathrm{M}=3.03, \mathrm{SD}=2.53)$ and upper performers $(\mathrm{M}=8.15, \mathrm{SD}=5.96)$ as they solved the puzzles; $\mathrm{t}(35)=-5.914$, $\mathrm{p}=.000$. There was not a significant difference in the number of add pass moves for lower performers $(\mathrm{M}=2.70, \mathrm{SD}=2.2)$ and upper performers $(\mathrm{M}=3.22, \mathrm{SD}=3.369)$ as they solved the puzzles; $\mathrm{t}(35)=-5.914, \mathrm{p}=.21$. There was not a significant difference in the number of rem pass moves for lower performers $(\mathrm{M}=1.18, \mathrm{SD}=1.23)$ and upper performers $(\mathrm{M}=1.34, \mathrm{SD}=1.91)$ as they solved the puzzles; $\mathrm{t}(35)=-.757$, $p=.45$. The results suggest that the types of moves different types of performers use matters in the successful solution to the puzzles, such as single, multi, and swap moves. However, the findings suggest that the more obscure moves, add and rem pass, were used less frequently overall, consequently no significant differences between the groups in the use of these types of moves was observed.

\section{Moves by Quarters of Puzzle Solving}

There was a significant difference in the number of moves in the first quarter for lower performers $(\mathrm{M}=7.74, \mathrm{SD}=13.87)$ and upper performers $(\mathrm{M}=12.90, \mathrm{SD}=23.71)$ as they solved the puzzles; $\mathrm{t}(45)=-3.107, \mathrm{p}=.003$. There was a significant difference in the number of moves in the second quarter for lower performers $(\mathrm{M}=7.82, \mathrm{SD}=13.27)$ and upper performers $(\mathrm{M}=12.96, \mathrm{SD}=22.94)$ as they solved the puzzles; $\mathrm{t}(45)=$ $-3.23, \mathrm{p}=.003$. There was a significant difference in the number of moves in the third quarter for lower performers $(\mathrm{M}=7.53, \mathrm{SD}=11.88)$ and upper performers $(\mathrm{M}=12.97$,
$\mathrm{SD}=21.28$ ) as they solved the puzzles; $\mathrm{t}(45)=-3.62, \mathrm{p}=.001$. There was a significant difference in the number of moves in the fourth quarter for lower performers $(\mathrm{M}=7.37, \mathrm{SD}=11.31)$ and upper performers $(\mathrm{M}=11.82, \mathrm{SD}=19.51)$ as they solved the puzzles; $\mathrm{t}(45)=-2.981, \mathrm{p}=.005$. The results suggest that upper performing players consistently used more moves across all quarters of play when compared to lower performing players.

\section{Puzzle Difficulty}

There was a significant difference in the number of moves used in the easy level of the puzzle lower performers $(\mathrm{M}=4.31$, $\mathrm{SD}=6.84)$ and upper performers $(\mathrm{M}=6.94, \mathrm{SD}=11.08)$ as they solved the puzzles; $\mathrm{t}(63)=-4.195, \mathrm{p}=.000$. There was a significant difference in the number of moves used in the medium level of the puzzle lower performers $(M=7.09$, $\mathrm{SD}=10.31)$ and upper performers $(\mathrm{M}=11.61, \mathrm{SD}=17.16)$ as they solved the puzzles; $\mathrm{t}(55)=-3.733, \mathrm{p}=.000$. There was a significant difference in the number of moves used in the hard level of the puzzle lower performers $(\mathrm{M}=12.10, \mathrm{SD}=17.36)$ and upper performers $(\mathrm{M}=20.58, \mathrm{SD}=30.838)$ as they solved the puzzles; $\mathrm{t}(59)=-4.436, \mathrm{p}=.000$. The results suggest that upper performing players consistently used more moves across difficulty levels of play when compared to lower performing players.

The results of the cross sectional analyses suggest that more moves, no matter the quarter, difficulty, and type influences higher scores and fewer violations. Based on these findings, it can be implied that upper performers constructed their knowledge differently from lower performers as they solved the electrical engineering puzzles.

\section{DISCUSSION}

It was hypothesized that learners would adapt to the game differently, although it was unknown as to how they would adapt. Findings show that the upper and lower level players of these games did differ in in their performances while solving the puzzles.

Constructing knowledge emanates from adapting learning and experiences to schema already embedded in the learner. The two sets of learners in the case of this electrical engineering game differed in multiple ways, suggesting a variance in constructed knowledge. The upper performance group was made more moves in each quarter, used more of each type of game, and no matter the difficulty, had more moves than their counterparts. The persistence in using more moves resulted in significantly more points, elimination of violations, and completion of the games. The upper performing learners showed a type of adaptation to the new environment that required altering schema as the games progressed. Although the upper performing learners used most types of moves more frequently, with the exception of the add pass and rem pass moves. The number of single, multi, and swap moves suggests a player who is willing to try and try again to achieve success with the game, as the game play progressed, they turned to alternate moves increasingly at each quarter, indicating that they were changing their original schema of single move only. The upper performers could be characterized as constructivist learners who are 
accommodators, learners who alter schema based on new information or experiences. They used the points, violations, and rewards to both achieve success in completion and change their perspective as the game progressed.

In contrast, the lower performers, who did not complete any of the games, seemed less motivated by points, and showed little concern for violations. Their constructed knowledge was evident by the fact that they did play, showed evidence of adjusting moves in various quarters of the game play, but made significantly fewer plays, resulting in non-completion. These players were using a constructed knowledge adaptation schema of assimilation. In this schema, the lower performing learners added new information, as evidenced by the use of all move types, albeit in smaller numbers than the upper performing group. However, in spite of the new information that was added, the lower level players did not use the information to change their schema, thereby never completing the games or using enough plays to create enough new information and experiences that would have led to success in the games.

\section{IMPLICATIONS FOR EDUCATION AND CONCLUSION}

Teachers, either those standing in front of a classroom, lecturing, handing out assignments, or game designers who design games with an educational purpose, are both challenged to recognize the need of both constructors of knowledge: assimilators and accommodators. If the information to be learned or skill to be developed are critical to the learners' advancement in a field or gains in important knowledge or concept development, teachers and game designers are challenged to find a way to guide the assimilator adaptive constructors of learning to include more accommodator characteristics in their knowledge construction. It is incumbent on teachers to reach out to these learners and tap into what motivates them to learn, and what incremental instructional frames would be necessary to engage these learners. Scaffolding techniques, related directly to the learners' gains would be critical to assure success. Piaget suggests that for learners to construct their knowledge in a balanced manner, it is important that both accommodation and assimilation types of thinking about learning occur. To accept one or the other is insufficient and it is incumbent on teachers to recognize this and find additional means by which to guide students through constructing their knowledge through both schema types.

Likewise, game designers, knowing what is necessary to complete games successfully could potentially design games so that the learner is prompted to use the appropriate number of moves, and with elegance, resulting in completion and potentially learning. A game of this nature would adapt to the learner when it is apparent the learner is abandoning the game, accepting their own schema as assimilators, rather than using the game information and experiences to change schema, applying the accommodation schema. The games can provide tips, clues, design choices, and alternative move strategies during the game play that guide players towards getting good solutions.

The results of the game play suggest that two distinct types of learners elected to play the game and in the case of the upper performers, finish it. Often in educational settings students not motivated by external rewards, such as points, grades, and lack of violations, require a teacher or game designer who is eager to reach all learners. This requires alternative perspectives and approaches to the learner. This requires extra time to get to know the learner, or in the case of the game designer, the consideration of alternative structures that serve to engage and envelop the lower performing student. If the material is important enough to learn, it behooves the classroom teacher as well as the game designer to consider the needs of both constructors of knowledge, the accommodator who can change schema, and the assimilator who goes through the experience and does not alter schema. Both learners, if guided to meld the two, can adapt, and learn.

\section{ACKNOWLEDGEMENTS}

We would like to thank the National Science Foundation for supporting this work with grant NSF CCF-1117800 and NSF CCF-1218656. We would like to acknowledge Emily Lofaro and Xiaozhong Luo for their contributions to this work. We would like to thank all the UNTANGLED players

\section{REFERENCES}

[1] J. Piaget and B. Inhelder, "The psychology of the child," Basic Books, 2008.

[2] C. S. Ang, E. Avni, and P. Zaphiris, "Linking pedagogical theory of computer games to their usability," International Journal on e-Learning, vol. 7, no. 3, pp. 533-558, 2008.

[3] S. E. Nielsen, "Third generation educational use of computer games," Journal of Educational Multimedia and Hypermedia, vol. 16, no. 3, pp. 263-281, 2007.

[4] A. Gopnik and H. M. Wellman, "Reconstructing constructivism: Causal models, Bayesian learning mechanisms, and the theory theory," Psychological Bulletin, vol. 138, vol. 6, pp. 1085, 2012.

[5] B. Hoge and F. Hughes, "The pedagogy behind the new century energy game: The potential for transformative PBL," Journal of Technology Integration in the Classroom, vol. 2, no. 3, pp. 11-22, 2010.

[6] W. C. Kriz, "A systemic-constructivist approach to the facilitation and debriefing of simulations and games," Simulation \& Gaming, vol. 41, no. 5, pp. 663-680, 2010.

[7] T Lainema, "Perspective making constructivism as a Meaning-Making structure for simulation gaming," Simulation \& Gaming, vol. 40, no. 1, pp. 48-67, 2009

[8] R. A. M. Rosario and G. R. Widmeyer, "An exploratory review of design principles in constructivist gaming learning environments," Journal of Information Systems Education, vol. 20, no. 3, pp. 289-300, 2009.

[9] W. H. Wu, H. C. Hsiao, P. L. Wu, and C. H. Lin, "Investigating the learning-theory foundations of game-based learning: A meta-analysis," Journal of Computer Assisted Learning, vol. 28, no. 3, pp. 265-279, 2012.

[10] S. S. Mansour and M. El Said, "Multi-players role-playing educational serious games: A link between fun and learning," International Journal of Learning, vol. 15, no. 11, pp. 229-239, 2009.

[11] C. D. Moore, C. A. Beshke, and C. H. Bohan, "Simulations and games in the civics classroom," Social Studies Research and Practice, vol. 9 , no. 2, pp. 77-88, 2014

[12] M. Papastergiou, "Online computer games as collaborative learning environments: Prospects and challenges for tertiary education," Journal of Educational Technology Systems, vol. 37, no.1, pp. 19-38, 2008.

[13] A. A. Razak and T. M. Connolly3min, "Using games-based learning: How it influences the learning experience and outcomes of primary school children," International Journal of Emerging Technologies in Learning (iJET), pp. 47-54, 2013.

[14] J. M. Applefield, R. Huber, and M. Moallem, "Constructivism in theory and practice: Toward a better understanding," The High School Journal, vol. 84, no. 2, pp. 35-53, 2000. 
[15] A. F. Brandon and A. C. All, "Constructivism theory analysis and application to curricula," Nursing Education Perspectives, vol. 31, no. 2, pp. 89-92, 2010

[16] D. H. Jonassen, "A constructivist's perspective on functional contextualism," Educational Technology Research and Development, vol. 54, no. 1, pp. 43-47, 2006.

[17] Y. Karagiorgi and L. Symeou, "Translating constructivism into instructional design: Potential and limitations," Educational Technology \& Society, vol. 8, no. 1, pp. 17-27, 2005.

[18] A. C. K. Leung, "Contextual issues in the construction of computer-based learning programs," Journal of Computer Assisted Learning, vol. 19, no. 4, pp. 501-516, 2003.

[19] J. Pascarella, "Confronting the challenges of critical digital literacy: An essay review critical constructivism: A primer," Educational Studies, vol. 43, no. 3, pp. 246-255, 2008.

[20] R. G. Saadé and D. Kira, "Effectiveness of an interactive application to assist learning: A test case," Journal of Information Systems Education, vol. 15, no. 4, pp. 357-363, 2004.

[21] V. M. Chieu, "An operational approach for building learning environments supporting cognitive flexibility," Educational Technology \& Society, pp. 32-46, 2007.

[22] J. G. Delia, "Constructivism and the study of human communication," Quarterly Journal of Speech, vol. 63, no. 1, pp. 66-83, 1977.

[23] B. Gholson and S. D. Craig, "Promoting constructive activities that support vicarious learning during computer-based instruction," Educational Psychology Review, vol. 18, no. 2, pp. 119-139, 2006.

[24] D. Giustini, "Utilizing learning theories in the digital age: From theory to practice," Journal of the Canadian Health Libraries Association/Journal de l'Association des bibliothèques de la santé du Canada, vol. 30, no. 1, pp. 19-25, 2014.

[25] T. Morgan, "Transformation through Interdependence: Learning to Collaborate," 1997.

[26] S. C. Goldstein et al., "PipeRench: A reconfigurable architecture and compiler," Computer, vol. 33, no. 4, pp. 70-77, 2000.

[27] L. Terry et al., "Harnessing Human Computation Cycles for the FPGA Placement Problem," ERSA, vol. 9, pp. 188-194, July 2009.

[28] L. Ahn and L. Dabbish, "Labeling images with a computer game," in Proc. the SIGCHI Conference on Human Factors in Computing Systems, ACM, pp. 319-326, 2004.

[29] L. Ahn, M. Blum, and J. Langford, "Telling humans and computers apart automatically," Communications of the ACM, pp. 56-60, 2004.

[30] L. Ahn et al., "Improving accessibility of the web with a computer game," in Proc. the SIGCHI Conference on Human Factors in Computing Systems, ACM, pp. 79-82, 2006.

[31] L. Ahn, R. Liu, and M. Blum, "Peekaboom: A game for locating objects in images," in Proc. the SIGCHI Conference on Human Factors in Computing Systems, ACM, pp. 55-64, April 2006.

[32] L. Ahn and L. Dabbish, "Designing games with a purpose," Communications of the ACM, vol. 51, no. 8 pp. 58-67, 2008.

[33] S. Cooper et al., "The challenge of designing scientific discovery games," in Proc. the Fifth International Conference on the Foundations of Digital Games, ACM, pp. 40-47, 2010.
[34] C. R. Sunstein, Infotopia: How Many Minds Produce Knowledge, Oxford University Press, 2006.

[35] J. Surowiecki, "The wisdom of crowds," Anchor, 2005.

[36] P. Michelucci, Handbook of Human Computation, Springer, 2013.

[37] G. Mehta et al., "Untangled: A game environment for discovery of creative mapping strategies," ACM Transactions on Reconfigurable Technology and Systems (TRETS), vol. 6, no. 3 p. 13, 2013

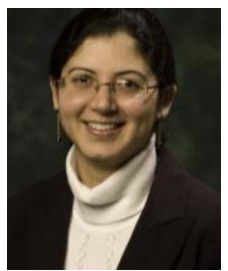

Gayatri Mehta is an associate professor in the Department of Electrical Engineering at the University of North Texas. She received her Ph.D in electrical and computer engineering from the University of Pittsburgh in 2009. Her research interests are broadly in the areas of reconfigurable computing, low-power VLSI design, system-on-a chip design, electronic design automation, embedded systems, portable/ wearable computing.

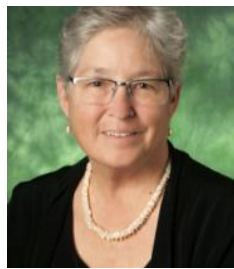

Jeanne Tunks is an associate professor in the College of Education, with specialization in curriculum and instruction and educational research. She currently serves as director of the Core Curriculum for the University of North Texas.

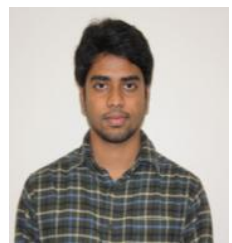

Kiran Sanagapaty did his master's in computer science and engineering from the University of North Texas. He worked in the Reconfigurable Computing Lab at UNT under the mentorship of Dr. Gayatri Mehta.

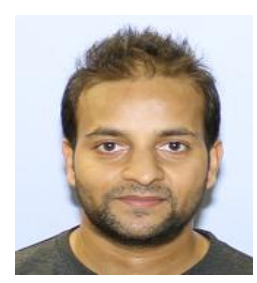

Alok Pal is working towards his master's degree in computer science and engineering at the University of North Texas. He works in the Reconfigurable Computing Lab at UNT under the mentorship of Dr. Gayatri Mehta.

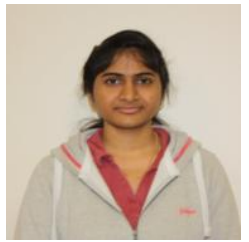

Rani Deepika Balavendran Joseph is currently working towards her $\mathrm{Ph}$. D in electrical engineering at the University of North Texas. She is working in the Reconfigurable Computing Lab at UNT under the mentorship of Dr. Gayatri Mehta. 